\title{
Hemostasis System Indices after Long-term Spaceflights
}

\author{
D. S. Kuzichkin, A. A. Markin, and B. V. Morukov \\ Institute of Biomedical Problems, Russian Academy of Sciences, Moscow, 123007 Russia \\ e-mail: andre_markine@mail.ru \\ Received March 1, 2010
}

DOI: $10.1134 / \mathrm{S} 0362119714070093$

Hemostasis was studied in six cosmonauts after long-term (178-199 days) spaceflights (SFs). We have revealed a post-SF reduction in activated partial thromboplastin time (aPTT), which is evidence of activation of the intrinsic pathway of coagulation. Antiplasmin (AP) activity decrease, protein C (PC) activity increase and fibrinogen concentration increase tendencies was observed. Changes in the investigated parameters were within the physiologically normal range.

The aim of this study was to estimate the functional activity of the main components of the hemostatic system after long-term SFs.

\section{METHODS}

The following hemostatic indices were determined in citrate plasma of six cosmonauts after long-term (178-199 days) SFs: blood coagulation parameters of the intrinsic (aPTT) and extrinsic (prothrombin time (PT), prothrombin index (PI), and international normalized ratio (INR)) pathways, as well as at its final stage (thrombin time (TT) and fibrinogen concentration); the activity of the anticoagulant enzymes (antithrombin III (AT III), PC, the concentration of plasminogen (PG), AP, as well as the content of fibrin formation products (soluble fibrin-monomer complexes (SFMCs)), and fibrin degradation products (Ddimer).

The biomaterial was sampled 45-30 days before the SF, as well as in the first and seventh day of the post-spaceflight period.

Investigations were performed with an automatic coagulometer Susmex CA-1500 (Japan) using commercial kits produced by Dade Behring (Germany). The SFMC content was determined by the paracoagulation method using the Tekhnologiya-Standart kits (Russia).

The data were treated by the methods of variance statistics using Student's $t$ test. Dixon's test was used to exclude individual values not belonging to the general sampled totality.

\section{RESULTS AND DISCUSSION}

The majority of the pre-SF background parameters were within the physiologically normal range. On the first post-SF day, we revealed a significant $10 \%$ increase in the fibrinogen concentration and a $27.8 \%$ reduction of aPTT compared with the preflight values. The trend towards a decrease in the activity of AP and an increase in the activity of PC was observed. No significant changes were detected in the time of extrinsic pathway of coagulation and at its final stage. The concentrations of PG, SFMC, D-dimer, as well as the activity of AT III, did not change significantly. All changes occurred within the limits of normal values.

On the seventh day of the post-SF period, there were no differences from the background values in any indices. The aPTT value returned to the level of its preflight values, exceeding the upper limit of normal values. The excessive activation of the intrinsic coagulation pathway was probably relieved during rehabilitation, due to the completion of the acute stress stage of readaptation to terrestrial conditions.

Thus, on the first day after a long-term SF, the activation of the intrinsic pathway of coagulation with increased fibrinogen concentration was observed, which indicated acute-phase responses, as well as an increasing trend of the activity of PC and a decreasing trend of the AP activity. These indices were observed to return to their preflight values on the seventh day of the post-SF period.

All the observed changes, excluding the aPTT values, were within the physiologically normal limits. They are obviously based on the action of physiological effects (specific blood flow characteristics in different segments of the vascular system, the level of hydratation, the development of a stress-response due to psycho-emotional stress), which were caused by a set of spaceflight-generated factors (staying in weightlessness, overloads at the initial and final SF stages), as well as the specific factors of readaptation to the terrestrial conditions. It is not excluded that intense physical loads aimed to prevent the unfavorable effects of long-term SFs may also affect the hemostatic system pointing to the necessity of monitoring prophylactic measures, including the use of laboratory diagnostics methods.

Translated by N. Tarasyuk 